\title{
REAL HYPERSURFACES OF AN ALMOST HYPERBOLIC HERMITIAN MANIFOLD
}

\author{
SUSHIL SHUKLA
}

\begin{abstract}
The purpose of the present paper is to study real hyper surfaces of an almost hyperbolic Hermitian manifold.
\end{abstract}

\section{Introduction}

Definition 1.1. Let us consider a differentiable manifold $M$ of class $C^{\infty}$ endowed with a tensor field $F$ of type $(1,1)$ such that

$$
\begin{gathered}
\widetilde{F}^{2}=I, \text { i.e. } \cdots \\
\widetilde{F}_{k}^{h} \widetilde{F}_{i}^{k}=\delta_{i}^{h},
\end{gathered}
$$

and

$$
g(F X, F Y)+g(X, Y)=0
$$

Then we say that $g$ is compatible with structure $F$ and $(F, g)$ is called almost hyperbolic Hermitian structure and the manifold $M$ with this structure is called almost hyperbolic Hermitian manifold.

Summation Convention: In the sequel, manifolds, tensor fields, connections and mappings we consider are assumed to be differentiable and of class $C^{\infty}$ unless otherwise stated and the indices $a, b, c, d, e, \ldots$ run over the range $\{1,2, \ldots 2 n+1\}$, the summation convention being used with respect to this system of indices.

Let there be given, on a manifold $M$ of odd dimension $2 n+l(\geq 3)$, a tensor field $f$ of type $(1,1)$, a vector field $\xi$ and a 1 -form $\theta$ satisfying

$$
f^{2}=I-\theta \otimes \xi, \quad f(\xi)=0, \quad \theta(f)=0, \quad \theta(\xi)=1,
$$

$I$ being the identity tensor field of type $(1,1)$, or

$$
f_{e}^{a} f_{b}^{e}=\delta_{b}^{a}-\theta \otimes \xi, \quad f_{e}^{a} \xi^{e}=0, \quad \theta_{e} f_{b}^{e}=0 \quad \theta_{e} \xi^{e}=1,
$$

Received July 17, 2008.

Key words and phrases. Hypersurface, almost hyperbolic Hermitian manifold, pseudo-conformal transformations, affine connection, hyperdistribution . 
$f_{b}^{a}, \xi^{a}$ and $\theta_{b}$ denoting components of $f, \xi, \theta$ respectively. Then the triple $(f, \xi, \theta)$ is called an almost para contact structure in $M$.

We define tensor fields $S$ of type $(1,2), G$ of type $(0,2), T$ of type $(0,2), P$ of type $(1,1)$ and $Q$ of type $(0,1)$ as those with components

$$
\begin{gathered}
S_{c b}^{a}=f_{c}^{e} \nabla_{e} f_{b}^{a}-f_{b}^{e} \nabla_{e} f_{c}^{a}-\left(\nabla_{c} f_{b}^{e}-\nabla_{b} f_{c}^{e}\right) f_{e}^{a}+\left(\nabla_{c} \theta_{b}-\nabla_{b} \theta_{b}\right) \xi^{a} \\
G_{c b}=f_{c}^{e}\left(\nabla_{e} \theta_{b}-\nabla_{b} \theta_{e}\right) \\
T_{c b}=G_{c b}-G_{c b}, \\
P_{b}^{a}=-\left[\xi^{e} \nabla_{e} f_{b}^{a}-\left(\nabla_{e} \xi^{a}\right) f_{b}^{e}+\left(\nabla_{b} \xi^{e}\right) f_{b}^{a}\right] \\
Q_{b}=-\left[\xi^{e} \nabla_{e} \theta_{b}+\left(\nabla_{b} \xi^{e}\right) \theta_{e}\right]
\end{gathered}
$$

respectively, where $\nabla$ denotes the operator of covariant differentiation with respect to an arbitrary symmetric affine connection in $M$. We easily see that these tensor fields are independent of the symmetric connection $\nabla$ used to define them. Then $S$ and $G$ are respectively called the torsion tensor and the Levi tensor of $(f, \xi, \theta)$. The following propositions are well known [4].

$\left(A_{1}\right) S=0$ implies $T=0, P=0$ and $Q=0$;

$\left(A_{2}\right) P=0$ implies $Q=0$.

When the tensor field $S$ vanishes identically, the almost para contact structure $(f, \xi, \theta)$ is said to be normal.

We now state an elementary lemma for later use. Let $V$ be a vector space over real number field with almost hyperbolic Hermitian structure $F$. That is, $F: V \rightarrow V$ is a linear transformation satisfying $F^{2}=I$ and $g(F X, F Y)+g(X, Y)=0$. Then $V$ is necessarily even-dimensional, say $\operatorname{dim} V=2 n+2(\geq 4)$. Take arbitrarily a $(2 n+l)$ -dimensional subspace $W$ of $V$. Then $F W$ is also $(2 n+1)$-dimensional. We can now state.

Lemma 1.1. Put $D=W \cap F W$ and $N=D-F W$. Then $F D=D, F N \subset W$, $V=W+F W, \operatorname{dim} D=2 n, N=\left(a x_{0}+y / a \in R, a \neq O, y \in D\right), x_{0}$ being a fixed element of $N$, and any element $x$ of $N$ is uniquely represented as $x=a x_{0}+y \quad(a \in R, y \in D)$.

The subset $N$ appearing in Lemma 1.1 has two connected components, each of which is homeomorphic to a Euclidean space of dimension $2 n+1$. The subset $T$ is called the affine normal space to $W$ in the vector space $V$ with al,ost hyperbolic Hermitian structure $F$. 


\section{Hypersurfaces of almost hyperbolic Hermitian manifold}

Let $M$ be a almost hyperbolic Hermitian manifold of real dimension $2 n+2(\geq 4)$ with almost hyperbolic Hermitian structure $F$, where $F$ is a tensor field of type $(1,1)$ in $M$ satisfying $\widetilde{F}^{2}=I$, i.e. ...

and

$$
\widetilde{F}_{k}^{h} \widetilde{F}_{i}^{k}=\delta_{i}^{h}
$$

$$
g(F X, F Y)+g(X, Y)=0
$$

$\widetilde{F}_{i}^{h}$ denoting components of $\widetilde{F}$.

Let there be given a hyper surface $M$ immersed in $\widetilde{M}$. For each point $P$ of $M$, denote the tangent space to $\widetilde{M}$ and that to $M$ at $P$ by $T_{P}(\widetilde{M})$ and $T_{P}(M)$ respectively. Then the subspace $D_{P}=T_{P}(M) \cap \widetilde{F} T_{P}(M)$ is $2 n$-dimensional and hence the correspondence $P \rightarrow D_{P}$ defines a distribution $D$ of dimension $2 n$ in $M$. Since $F D=D$, we can define a tensor field $J$ of type $(1,1)$ in $D$ by $J X=\widetilde{F} X, X$ being an arbitrary vector field belonging to $D$. Then $\widetilde{F}^{2}=I$ implies $J^{2}=I_{D}$, where $I_{D}$ denotes the identity tensor field of type $(1,1)$ in $D$. Thus the $D$ is called a hyperdistribution with almost hyperbolic Hermitian structure $J$ in $M$ and said to be induced in $M$ from $\widetilde{F}$ by the immersion [3].

Since the tangent space $T_{P}(\widetilde{M})$ is a vector space with almost hyperbolic Hermitian structure $\widetilde{F}$, by Lemma 1.1 the subspace $T_{P}(M)$ of $T_{P}(\widetilde{M})$ has its affine normal space $N_{P}$. We call $N=\bigcup_{P \in M} N_{P}$ the affine normal bundle to the hyper surface $M$.

Since $N_{P}$ has two connected components, each of which is homeomorphic to a Euclidean space, $N$ has a global cross-section if $M$ is orientable.

Let $\bar{U}$ be a coordinate neighborhood of $\widetilde{M}$ such that any connected component $U$ of $\bar{U} \cap M$ is a coordinate neighborhood of $M$. In the sequel by $U$ we mean such a coordinate neighborhood of $M$. Take a local cross-section $C$ of the affine normal bundle $N$ over $U$ and call it a local affine normal to $M$ in $U$. Then by Lemma $1.1 \widetilde{F} C$ is tangent to $M$ in $U$ and hence

$$
\xi=\widetilde{F} C
$$

is a non-vanishing vector field in $U$. Next, for any vector field $X$ in $M$, we can decompose $\widetilde{F} X$ uniquely as

$$
\widetilde{F} X=f X+\theta(X) C,
$$

where $f X$ is tangent to $M$. Thus $f$ and $\theta$ are a tensor field of type $(1,1)$ and a 1 -form in $U$ respectively. Applying $\widetilde{F}$ to $(2.3)$ and using $\widetilde{F}_{2}=I$, we find $X=\left(f^{2} X+\theta(X) \xi\right)+\theta(f X) C$, which implies

$$
f^{2}=I-\theta \otimes \xi, \quad \theta(f)=0 .
$$

If we put $X=\xi$ in (2.3), we obtain $\widetilde{F} \xi=f(\xi)+\theta(\xi) C$. On the other hand (2.2) gives $\widetilde{F} \xi=C$. Hence we get

$$
f(\xi)=0, \quad \theta(\xi)=l .
$$


Equations (2.4) and (2.5) show that the triple $(f, \xi, \theta)$ is an almost contact structure in $U$, which is called an almost contact structure induced in $M$ by an affine normal $C$ in $U$. A vector field $X$ in $M$ belongs to $D$ if and only if $\widetilde{F} X$ belongs to $D$. Thus, because of (2.3), $X$ belongs to $D$ if and only if $\theta(X)=0$. Hence the distribution $D$ is defined by $\theta=0$ in $U$. Therefore the almost contact structure $(f, \xi, \theta)$ is associated with the hyperdistribution $D$ with complex structure [3].

We now take another affine normal $\bar{C}$ to $M$ in $U$. Then by Lemma 1.1 we have

$$
\bar{C}=-\frac{1}{\alpha}(C+A),
$$

where $\alpha$ is a non-vanishing function and $A$ a vector field being tangent to $M$ and belonging to $D, \alpha$ and $A$ being defined in $U$. Thus we have

$$
\bar{f}=-f+\theta \otimes \xi, \quad \bar{\xi}=-\frac{1}{\alpha}(\xi-f A), \quad \bar{\theta}=\alpha \theta,
$$

where $(\bar{f}, \bar{\xi}, \bar{\theta})$ is the almost contact structure induced in $M$ by (2.3) and (2.5), $C$ being replaced by $\bar{C}$. The change $(2.7)$ of almost contact structures has been discussed in [3] and is called a change of almost contact structures associated with $D$.

\section{Induced affine connections}

We now assume that the ambient manifold $\widetilde{M}$ is a complex manifold of complex dimension $n+1(\geq 2)$ with almost hyperbolic Hermitian structure $\widetilde{F}$. It is well known that there is a symmetric affine connection $\widetilde{\nabla}$ satisfying $\widetilde{\nabla} \widetilde{F}=0$, i.e.

$$
\widetilde{\nabla}_{j} \widetilde{F}_{i}^{h}=0
$$

In the sequel we fix this affine connection $\widetilde{\nabla}$.

Consider a real hypersurface $M$ immersed in $\widetilde{M}$ and a coordinate neighborhood $U$ of $M$ such that $U$ is a connected component of $\bar{U} \cap M, \bar{U}$ being a coordinate Neighborhood of $\widetilde{M}$. Let $\left(x^{h}\right)$ and $\left(y^{a}\right)$ be coordinates in $\bar{U}$ and in $U$ respectively. We assume that $M$ is represented in $\bar{U}$ by

$$
x^{h}=x^{h}\left(y^{a}\right) .
$$

Take an affine normal $C$ to $M$ in $U$ and put

$$
B_{b}^{h}=\partial x^{h} / \partial y^{a}
$$

in $U$. Then $B_{b}=B_{b}^{h} \partial / \partial x^{h}$ and $C=C^{h} \partial / \partial x^{h}$ form an affine $(2 n+2)$-frame along $U$.

Thus on putting

$$
\frac{B_{i}^{a}}{C_{i}}=-\left(B_{b}^{h}, C^{h}\right)^{-1}
$$

we have

$$
B_{b}^{i} B_{i}^{a}=\delta_{b}^{a}, \quad B_{i}^{a} C^{i}=0, \quad C_{i} C^{i}=1
$$




$$
B_{e}^{h} B_{i}^{e}+C^{h} C_{i}=-\delta_{i}^{h} .
$$

Thus $B^{a}=B_{i}^{a} \partial x^{i}$ and $C=C_{i} \partial x^{i}$ form a coframe dual to $\left\{B_{b}, C\right\}$ along $U$.

The affine connection $\nabla$ induced in $U$ from $\widetilde{T}_{j i}^{h}$ with respect to the affine normal $C$ has, by definition, components given by

$$
T_{c b}^{a}=\left(\partial_{c} B_{b}^{h}+\widetilde{T}_{j i}^{h} B_{c}^{j} B_{b}^{i}\right) B_{h}^{a}
$$

where $\partial_{b}=\partial / \partial y^{b}$ and $\widetilde{T}_{j i}^{h}$ denote components of $\widetilde{\nabla}$ in $\widetilde{U}$. Since $\widetilde{\nabla}$ is symmetric, i.e. ... since $\widetilde{T}_{j i}^{h}=\widetilde{T}_{i j}^{h} \nabla$ is also symmetric, i.e. . . . T $T_{c b}^{a}=T_{b c}^{a}$. Thus if we define the so- called vander Waerden- Bortolotti covariant derivative of $B_{b}^{h}$ along $M$ by

$$
\nabla_{c} B_{b}^{h}=-\partial_{c} B_{b}^{h}-\widetilde{T}_{j i}^{h} B_{c}^{j} B_{b}^{i}+T_{c b}^{a} B_{a}^{h}
$$

in $U$, then we have $\left(\nabla_{c} B_{b}^{h}\right) B_{b}^{a}=0$, which shows that $\nabla_{c} B_{b}^{h}$ is of the form

$$
\nabla_{c} B_{b}^{h}=h_{c b} C^{h},
$$

where $h_{c b}$ are defined by

$$
h_{c b}=h_{b c}=\partial_{c} B_{b}^{h}+\left(\widetilde{T}_{j i}^{h} B_{c}^{J} B_{b}^{i}\right) C_{h}
$$

and are called components of the covariant second fundamental tensor $h$ of $M$ with respect to the affine normal $C, h$ being of type $(0,2)$.

Differentiating $B_{b}^{h} B_{h}^{a}=-\delta_{b}^{a}$ covariantly along $M$ and using (3.8) and $C_{h} B_{h}^{a}=0$ we find $B_{b_{c b}}^{h}\left(\nabla_{c} B_{h}^{a}\right)=0$ from which

$$
\nabla_{c} B_{i}^{a}=-H_{c}^{a} C_{i}
$$

where $\nabla_{c} B_{i}^{a}$ are defined by

$$
\nabla_{c} B_{i}^{a}=-\partial_{c} B_{i}^{a}-\widetilde{T}_{j i}^{h} B_{c}^{j} B_{h}^{a}-T_{c b}^{a} B_{i}^{b}
$$

in $U$ and $H_{c}^{a}$ by

$$
H_{c}^{a}=\left(\partial_{c} B_{b}^{h}+\widetilde{T}_{j i}^{h} B_{c}^{j} C^{i}\right) B_{h}^{a} .
$$

The $H_{c}^{a}$ are called components of the mixed second fundamental tensor $H$ of $M$ with respect to the affine normal $C$ in $U, H$ being of type (1.1).

We next differentiate $B_{i}^{a} C^{i}=0$ covariantly along $M$ and use (3.10). Then we obtain $H_{c}^{a}-B_{i}^{a}\left(\nabla_{c} C^{i}\right)=0$ from which

$$
\nabla_{c} C^{h}=H_{c}^{a} B_{a}^{h}-l_{c} C^{h}
$$

where $l_{c}$ are defined by

$$
l_{c}=\left(\partial_{c} C^{h}+\widetilde{T}_{j i}^{h} B_{c}^{j} C^{i}\right) C_{h}
$$


and $\nabla_{c} C^{h}$ by

$$
\nabla_{c} C^{h}=\partial_{c} C^{h}+\widetilde{T}_{j i}^{h} B_{c}^{j} C^{i}
$$

in $U$. The $l_{c}$ are called components of the third fundamental tensor $l$ of $M$ with respect to the affine normal $C$ in $U, l$ being of type $(0,1)$. The $l$ gives a linear connection in the one-dimensional vector bundle

$$
\bigcup_{p \in U}\left\{a C_{P} / a \epsilon R\right\} \quad \text { over } U .
$$

Finally, differentiating $B_{b}^{i} C_{i}=0$ covariantly along $M$ and using (3.8), $C^{i} B_{i}^{a}=0$ and $C^{i} C_{i}=1$, we find $l_{c}-C^{i}\left(\partial_{c} C_{i}\right)=0$, from which

$$
\nabla_{c} C_{i}=h_{c e}+l_{c} C_{i}
$$

where $\nabla_{c} C_{i}$ are defined in $U$ by

$$
\nabla_{c} C_{i}=\partial_{c} C_{i}+\widetilde{T}_{j i}^{h} B_{c}^{j} C_{h} .
$$

Equations (3.8) and (3.10) are those of Gauss for the real hypersurface $M$ and equations (3.11) and (3.13) are those of Weingarten for $M$.

Consider a vector field $X=X^{h} \partial / \partial x^{h}$ tangent to $M$. Then we have $X^{h}=X^{a} B_{a}^{h}$ Thus using (3.8), we have

$$
\nabla_{c} X^{h}=-\left(\partial_{c} X^{a}\right) B_{a}^{h}-h_{c b} X^{b} C^{h},
$$

where we have put in $U$

$$
\nabla_{c} X^{h}=\partial_{c} X^{h}+\widetilde{T}_{j i}^{h} B_{c}^{j} X^{i}, \quad \nabla_{c} X^{a}=\partial_{c} X^{a}+T_{c b}^{a} X^{b} .
$$

Let $(f, \xi, \theta)$ be the almost contact structure induced in $M$ by the affine normal $C$ to $M$ in $U$. Then (2.2) and (2.3) can be written as

$$
\begin{aligned}
\widetilde{F}_{i}^{h} C^{i} & =\xi^{b} B_{b}^{h}, \\
\widetilde{F}_{i}^{h} B_{b}^{i} & =f_{b}^{a} B_{a}^{h}-l_{b} C^{h}
\end{aligned}
$$

respectively. Applying $\nabla_{c}$ to $(3.20)$ and using $\nabla_{c} \widetilde{F}_{i}^{h}=B_{c}^{j} \widetilde{\nabla}_{j} \widetilde{F}_{i}^{h}=0$, we obtain

$$
h_{c b}\left(f^{a} B_{a}^{h}\right)=\left(\nabla_{c} f_{b}^{a}\right) B_{a}^{h}+h_{c e} f_{b}^{e} C^{h}-\left(\nabla_{c} \theta_{b}\right) C^{h}+\left(H_{c}^{a} B_{a}^{h}-l_{c} C^{h}\right),
$$

where we have used (2.2), (2.3) with $X=B_{b}$ (3.16) and (3.17). Thus we obtain

$$
\begin{aligned}
& \nabla_{c} f_{b}^{a} h_{c b} \xi^{a}-H_{c}^{a} \theta_{b}, \\
& \nabla_{c} \theta_{b}=h_{c e} f_{b}^{e}+l_{c} \theta_{b} .
\end{aligned}
$$

Next, applying $\nabla_{c}$ to (3.19), we have in a similar way as above from which

$$
\begin{aligned}
\nabla_{c} \xi^{a} & =f_{e}^{a} H_{c}^{e}-l_{c} \xi^{a} \\
H_{c}^{e} \theta_{e} & =-h_{c e} \xi^{e}
\end{aligned}
$$


Substituting (3.21), (3.22) and (3.23) into (1.3) and using (3.24), we obtain

$$
\begin{aligned}
S_{c b}^{a} & =\left(-H_{e}^{a} f_{c}^{e}+f_{e}^{a} H_{c}^{e}-l_{c} \xi^{a}\right) \theta_{b}+\left(-H_{e}^{a} f_{b}^{e}+f_{e}^{a} H_{b}^{e}-l_{b} \xi^{a}\right) \theta_{c} \\
G_{c b} & =h_{c b}+f_{c}^{e} f_{b}^{d} h_{e d}-h_{b e} \xi^{e} \theta_{c}+f_{c}^{e} I_{e} \theta_{b} \\
Q_{b} & =-l_{b}+\left(I_{e} \xi^{e}\right) \theta_{b}+h_{e d} \xi^{e} f_{b}^{d}
\end{aligned}
$$

When a hyperdistribution $D$ with almost hyperbolic Hermitian structure $J$ is given on a manifold of odd dimension and when $S_{c b}^{a} \equiv 0,\left(\bmod \theta_{c}, \theta_{b}\right)$ is satisfied for an almost para contact structure $(f, \xi, \theta)$ associated with $D$, the $D$ is said to be torsionless. Thus we have from (3.25).

Proposition 3.1. For any real hypersurface $M$ of an almost hyperbolic Hermitian manifold the induced hyperdistribution $D$ of $M$ with almost hyperbolic Hermitian structure $J$ is always torsionless.

Equations (3.26) imply

Proposition 3.2. For any real hypersurface $M$ of a almost hyperbolic Hermitian manifold, the Levi-tensor $G$ of an almost para contact structure $(f, \xi, \theta)$ induced in $M$ has components of the form

$$
G_{c b}=h_{c b}+f_{c}^{e} f_{b}^{d} h_{e d} \quad\left(\bmod \theta_{c}, \theta_{b}\right)
$$

in $U$, when an affine normal $C$ to $M$ is given in a coordinate neighborhood $U$ of $M$.

Proposition 3.2 implies that

$$
g(X, Y)=g(Y, X), \quad g(J X, J Y)+g(X, Y)=0
$$

for any vector fields $X$ and $\mathrm{Y}$ belonging to the hyperdistribution $D$ with hyperbolic RAC Structure J. Equations (3.25) imply.

Proposition 3.3. Let $(f, \xi, \theta)$ be an almost para contact structure induced on a real hypersurface $M$ of an almost hyperbolic Hermitian manifold by giving an affine normal $C$ to $M$ in a coordinate neighborhood $U$ of $M$. Then $(f, \xi, \theta)$ is normal if and only if

$$
-H_{e}^{a} f_{b}^{e}+f_{e}^{a} H_{b}^{e}-l_{b} \xi^{a} \equiv 0, \quad\left(\bmod \theta_{c}, \theta_{b}\right) .
$$

We take another affine normal $\bar{C}$ to $M$ in $U$ and assume $\bar{C}$ is given by (2.6). Denote by $\bar{\nabla}, \bar{l}, \bar{h}$ and $\bar{H}$ respectively the induced affine connection, the third fundamental tensor, the covariant and the mixed second fundamental tensors of $M$ in $U$, which are determined by (3.6), (3.14), (3.9) and (3.12) in terms of $\bar{C}$.

Then components $\bar{T}_{c a}^{a}$ of $\bar{\nabla}, \bar{h}_{c b}$ of $\bar{h}, \bar{H}_{b}^{a}$ of $\bar{H}$ and $\bar{l}_{b}$ of $\bar{l}$ are respectively given by

$$
\begin{aligned}
\bar{T}_{c b}^{a} & =T_{c b}^{a}+h_{c b} A^{a}, \quad \bar{h}_{c b}=\alpha h_{c b} \\
\bar{H}_{b}^{a} & =-\frac{1}{\alpha}\left[H_{b}^{a}+\nabla_{b} A^{a}-\left(l_{b}+h_{b e} A^{e}\right) A^{a}\right], \\
\bar{l}_{b} & =-\left(l_{b}+h_{b e} A^{e}\right)+\nabla_{b} \log |\alpha|,
\end{aligned}
$$


where $\alpha$ is a non-vanishing function and $A=A^{a} B_{a}^{h} \partial / \partial x^{h}$ is a vector field belonging to $D$, both being defined in $U$. To obtain (3.30), we have used (2.6), $\bar{B}_{b}^{h}=B_{b}^{h}$ and

$$
\bar{B}_{i}^{a}=B_{i}^{a}+A^{a} C_{i}, \quad \bar{c}_{i}=C_{i},
$$

where

$$
\left(\frac{\bar{B}_{i}^{a}}{\bar{C}_{i}}\right)=-\left(\bar{B}_{a}^{h}, \bar{C}^{h}\right)^{-1} .
$$

Proposition 3.2 and $\bar{h}_{c b}=\alpha h_{c b}$ appearing in (3.30) imply the following well known Proposition $[1,2,3,5]$ :

Proposition 3.4. Let $(f, \xi, \theta)$ and $(\bar{f}, \bar{\xi}, \bar{\theta})$ be two almost para contact structures induced on a real hypersurface $M$ and assume that they are related to each other by (2.7). Then

$$
\bar{G}_{c b}=\alpha G_{c b} \quad\left(\bmod \theta_{c}, \theta_{b}\right)
$$

$\alpha$ being a non-vanishing function, where $\bar{G}_{c b}$ and $G_{c b}$ are respectively components of the Levi tensors of $(f, \xi, \theta)$ and $(\bar{f}, \bar{\xi}, \bar{\theta})$.

Proposition 3.4 shows that the restriction $G_{D}$ of the Levi tensor $G$ to $D$ is determined up to a non-vanishing factor. Thus $G_{D}$ is sometimes called the Levi tensor of the induced hyper distribution $D$ with almost hyperbolic Hermitian structure. When $G$ is of the maximum rank $2 n$ everywhere in $M$, the real hyper surface $M$ is said to be nondegenerate. By P3.1, for any real hyper surface $M$ of an almost hyperbolic Hermitian manifold the hyper distribution $D$ of $M$ with almost hyperbolic Hermitian structure is torsion less. This fact means that any real hyper surface $M$ admits a pseudo-conformal structure when $M$ is non-degenerate $[1,2,5]$.

\section{Pseudo-conformal mappings}

Let $M$ and ' $M$ be two manifolds admitting hyperdistributions $D$ and ' $D$ with almost hyperbolic Hermitian structures $J$ and ' $J$ respectively. Assume that there is a homeomorphism $\Phi: M \rightarrow^{\prime} M$ such that, for any vector field $X$ belonging to $D, \Phi^{*} X$ belongs to ${ }^{\prime} D$ and $\Phi^{*} J={ }^{\prime} J \Phi^{*}$, where $\Phi^{*}$ denotes the differential of $\Phi$. Then $\Phi: M \rightarrow^{\prime} M$ is called a pseudo-conformal mapping $[3,5]$.

Let $\widetilde{\Phi}: \widetilde{M} \rightarrow \widetilde{M}$ be a holomorphic transformation of the ambient almost hyperbolic Hermitian manifold $\widetilde{M}$ with almost hyperbolic Hermitian structure $\widetilde{F}$. Then $\widetilde{\Phi}^{*} \widetilde{F}=\widetilde{F} \widetilde{\Phi}^{*}$, where $\widetilde{\Phi}^{*}$ denotes the differential of $\widetilde{\Phi}$. Consider real hypersurfaces $M$ and ' $M$ immersed in $\widetilde{\Phi}$ and assume $\widetilde{\Phi}(M)={ }^{\prime} M$. Denote by $\Phi: M \rightarrow{ }^{\prime} M$ the restriction of $\widetilde{\Phi}$ to $M$. Then $\Phi$ is a homeomorphism and is called the mapping induced from $\widetilde{\Phi}$. Let $D$ and ' $D$ be the hyperdistributions with almost hyperbolic Hermitian structure induced in $M$ and ' $M$ reservedly. Denote by $J$ and ' $J$ the almost hyperbolic Hermitian structures induced in $D$ and ${ }^{\prime} D$ respectively. Then we can easily verify that $\Phi^{*} X$ belongs to ${ }^{\prime} D$ whenever $X$ belongs to $D$ and that $\Phi^{*} J=^{\prime} J \Phi^{*}$. Thus $\Phi: M \rightarrow^{\prime} M$ is a pseudo-conformal mapping. Hence we have the following proposition: 
Proposition 4.1. Any holomorphic transformation of the ambient $\widetilde{\Phi}: \widetilde{M} \rightarrow \widetilde{M}$ of the ambient almost hyperbolic Hermitian manifold induces a pseudo-conformal mapping $\Phi: M \rightarrow{ }^{\prime} M$, where $M$ and ${ }^{\prime} M$ are real hypersurfaces in $\widetilde{M}$ such that ${ }^{\prime} M=\widetilde{\Phi}(M)$.

Let $\widetilde{\Phi}: \widetilde{M} \rightarrow \widetilde{M}$ and $\Phi: M \rightarrow^{\prime} M$ be taken as above. If we take an affine normal $C$ to $M$ in a coordinate neighborhood $U$ of $M$, then $\widetilde{C}=\Phi^{*}(C)$ is also an affine normal to ${ }^{\prime} M$ in ' $U=\Phi(U)$ because of $\widetilde{\Phi} * \widetilde{F}=\widetilde{F} \Phi^{*}$. Thus, taking an affine normal ' $C$ to ' $M$ in ${ }^{\prime} U$, we get because of $(2.6)$

$$
\bar{C}=-\frac{1}{\alpha}\left({ }^{\prime} C+A\right)
$$

in ' $U$, where $\alpha$ is a non-vanishing function and $A$ a vector field belonging to ' $D$, both being defined in ' $U$. Let $(f, \xi, \theta)$ be the almost para contact structure induced in $M$ by the affine normal $C$ to $M$ in $U$. Let $(\widetilde{f}, \widetilde{\xi}, \widetilde{\theta})$ be the almost para contact structure induced in ' $M$ by the affine normal ' $C$ to ${ }^{\prime} M$ in ' $U$. Then putting

$$
\bar{f}=\Phi^{*} f\left(\Phi^{*}\right)^{-1}, \quad \bar{\xi}=\Phi \xi, \quad \bar{\theta}=\theta(\Phi)
$$

we see that $(\tilde{f}, \widetilde{\xi}, \widetilde{\theta})$ is an almost para contact structure associated with 'D in 'U. Thus, taking account of (2.7), we have from (4.1)

$$
\bar{f}=-^{\prime} f+^{\prime} \theta \otimes A, \quad \bar{\xi}=-\frac{1}{\alpha}\left(\xi-^{\prime} f A\right), \quad \bar{\theta}=\alpha^{\prime} \theta .
$$

In general, the following proposition prevails:

Proposition 4.2. For a homeomorphism $\Phi: M \rightarrow{ }^{\prime} M$ of a manifold $M$ admitting a hyperdistributwn with almost hyperbolic Hermitian structure onto another ' $M,(4.3)$ is a necessary and sufficient condition for $\Phi: M \rightarrow{ }^{\prime} M$ to be a pseudo-conformal mapping.

\section{Infinitesimal pseudo-conformal transformations}

Let $X$ be a vector field on a manifold $M$ admitting a hyperdistribution $D$ with almost hyperbolic Hermitian structure and assume that any local transformations $\Phi_{t}(-\epsilon<t<$ $\epsilon, \epsilon>0)$ of $M$ generated by $X$ are always pseudo-conformal transformations. Then $X$ is called an infinitesimal pseudo-conformal transformation or simply a pseudo- conformal vector field in $M$. Let $(f, \xi, \theta)$ be an almost para contact structure associated with $D$ in a coordinate neighborhood $U$. Then we have the following lemma for a manifold admitting a hyperdistribution with almost hyperbolic Hermitian structure:

Proposition 5.1. In a real hypersurface $M$ of an almost hyperbolic Hermitian manifold, a vector field $X$ is pseudo-conformal if and only if $X$ satisfies

$$
L_{X} f=\theta \otimes V, \quad L_{X} \xi=a \xi+f V, \quad L_{X} \theta=a \theta,
$$

where $a$ is a function and $V$ a vector field belonging to $D$, both being defined in $U$. 
It is known that a pseudo-conformal vector field $X$ in $M$ vanishes identically if $X$ belongs to $D$, where $D$ is assumed to be torsionless and non-degenerate. On the other hand, by Proposition 3.1, for any real hypersurface $M$ of an almost hyperbolic Hermitian manifold $M$ the induced hyperdistribution $D$ of $M$ is always torsionless. Thus we have

Proposition 5.2. Let $M$ be a non-degenerate real hypesurface of an almost hyperbolic Hermitian manifold $\widetilde{M}$. A pseudo-conformal vector field $X$ in $M$ vanishes identically if $X$ belongs to the induced hyperdistribution $D$ with almost hyperbolic Hermitian structure.

Consider a real hypersurface $M$ of an almost hyperbolic Hermitian manifold $\widetilde{M}$ with almost hyperbolic Hermitian structure $\widetilde{F}$. Let a holomorphic vector field $\widetilde{X}$ in $\widetilde{M}$ be tangent to $M$. Then, since $X$ is holomorphic, $X$ satisfies

$$
\widetilde{F}_{k}^{h} \widetilde{\nabla}_{i} \widetilde{X}^{k}=\widetilde{\nabla_{k}} \widetilde{X}^{k} \widetilde{F}_{i}^{k}
$$

On the other hand, since $\widetilde{X}$ is tangent to $M$, we have along $M$

$$
\widetilde{X}^{h}=X^{a} B_{a}^{h} .
$$

Transvection of (5.2) with $B_{b}^{i}$ gives

$$
\widetilde{F}_{k}^{h}\left(\widetilde{\nabla}_{i} \widetilde{X}^{k}\right)=\left(\widetilde{\nabla}_{k} \widetilde{X}^{h}\right) \widetilde{F}_{i}^{k} B_{b}^{i}
$$

which is equivalent to

$$
\left[-\left(\nabla_{b} X^{e}\right) f_{e}^{a}+\left(\nabla_{e} X^{a}\right) f_{b}^{e}+h_{b e} X^{e} \xi^{a}\right] B_{a}^{h}-\left[\left(\nabla_{b} X^{e}\right) \theta_{e}-f_{b}^{e} h_{e d} X^{d}\right] C^{h}=\theta_{b}\left(C^{k} \widetilde{\nabla}_{k} \tilde{X}^{h}\right)
$$

because of (3.18), (3.19), (3.20) and (5.3), where $(f, \xi, \theta)$ almost para contact structure induced in each coordinate neighborhood $U$ of $M$ by fixing an affine normal $C$ to $M$ in $U$. Next, transvection of (5.2) with $C^{i}$ gives

$$
C^{i} \widetilde{\nabla}_{i} \widetilde{X}^{h}=-\xi^{c}\left[\left(\nabla_{c} X^{e}\right) f_{e}^{a}-h_{c e} X^{e} \xi^{a}\right] B_{a}^{h}-\left[\xi^{c}\left(\nabla_{c} X^{e}\right) \theta_{e}\right] C^{k}
$$

because of (3.18), (3.19), (3.20) and (5.3). Substituting (5.6) into (5.5), we have

$$
\begin{aligned}
&\left.-\left(\nabla_{i} X^{e}\right) f_{e}^{a}+\left(\nabla_{e} X^{a}\right) f_{b}^{e}+h_{b e} X^{e} \xi^{a}\right]=\theta_{b}\left[-\xi^{c}\left(\nabla_{c} X^{e}\right) f_{e}^{a}+h_{c e} \xi^{c} X^{e} \xi^{a}\right] \\
&\left(\nabla_{b} X^{e}\right) \theta_{e}-f_{b}^{e} h_{e d} X^{d}=\theta_{b}\left(\nabla_{c} X^{e}\right) \xi^{c} \theta_{e}
\end{aligned}
$$

which reduce respectively to

$$
L_{X} f_{b}^{a}=\theta_{b} V^{a}, \quad L_{X} \theta_{b}=a \theta_{b}
$$

where we have put

$$
\begin{aligned}
V^{a} & =H_{e}^{a} X^{e}+h_{c e} \xi^{c} X^{e} \xi^{a}-\xi\left(\nabla_{c} X^{e}\right) f_{e}^{a}, \\
a & =\left(\nabla_{c} X^{e}\right) \xi^{c} \theta_{e}-l_{e} X^{e} .
\end{aligned}
$$


Thus, taking account of (3.24), we see easily that $\theta_{e} V^{e}=0, \ldots$ that $V^{a}$ are components of a vector field $\mathrm{V}$ belonging to the induced hyperdistribution $D$ of $M$. Next, the identities $\theta_{b} \xi^{b}=1$ and $f_{b}^{a} \xi^{b}=0$ imply respectively

$$
\left(L_{X} \theta_{b}\right) \xi^{b}+\theta_{b}\left(L_{X} \xi^{b}\right)=0, \quad \text { and }\left(L_{X} f_{b}^{a}\right) \xi^{b}+f_{b}^{a}\left(L_{X} \xi^{b}\right)=0 .
$$

Substituting (5.8) into these equations, we obtain

$$
L_{X} \xi^{b}=a \xi^{a}+f_{e}^{a} V^{e} .
$$

Consequently, we have (5.1) from (5.8) and (5.10). Thus we have the following proposition:

Proposition 5.3. Let $M$ be a real hypersurface immersed in an almost hyperbolic Hermitian manifold. If a holomorphic vector field $\widetilde{X}$ in $\widetilde{M}$ is tangent to $M$, then the restriction $X$ of $\widetilde{X}$ to $M$ is a pseudo-conformal vector field in $M$.

Let $(f, \xi, \theta)$ be an almost para contact structure induced in a coordinate neighborhood $U$ of $M$ and assume that $\xi$ is a pseudo-conformal vector field in $U$. Then $(f, \xi, \theta)$ is said to be regular [5]. If this is the case, (5.1) implies

$$
P_{b}^{a}=L_{\xi} f_{b}^{a}=0,
$$

because $L_{\xi} \xi=0$ and (5.1) gives $V=0$ and $a=0$. Therefore (3.25) and (3.28) implies

$$
-H_{e}^{a} f_{b}^{e}+f_{e}^{a} H_{b}^{e}-l_{b} \xi^{a} \quad\left(\bmod \theta_{b}\right)
$$

if and only if $(f, \xi, \theta)$ is regular.Thus we have, from Proposition 3.3.

Proposition 5.4. In a real hypersurface $M$ immersed in an almost hyperbolic Hermitian manifold, an induced almost para contact structure of $M$ is normal if and only if it is regular.

Proposition 5.3 is however a consequence of Proposition 3.1.

Let $\left(Z^{\lambda}\right)$ be a system of complex coordinates in a coordinate neighborhood $\widetilde{U}$ of the ambient almost hyperbolic Hermitian manifold $M$. Then we have

Proposition 5.5. The condition (5.2) is equivalent to each of the following conditions

$$
\begin{gathered}
\frac{\partial \tilde{X}^{\lambda}}{\partial \bar{z}^{\mu}}=0 \\
d \widetilde{X}^{\lambda} \wedge d z^{1} \wedge \cdots \wedge d z^{n+1}=0
\end{gathered}
$$

It is easily verified that condition (5.8) is equivalent to (5.7) which is equivalent to (5.5) and hence to (5.4). Thus we have 
Proposition 5.6. Condition (5.8) is equivalent to (5.4) or to

$$
d \widetilde{X}^{\lambda} \wedge d\left(z^{1} \circ i\right) \wedge \cdots \wedge d\left(z^{n+1} o i\right)=0
$$

where $i: M \rightarrow \widetilde{M}$ is the immersion of $M$.

Let there be given a vector field $X$ in $M$ and put $\widehat{X}^{h}=X^{a} B_{a}^{h}$ where $X^{a}$ are components of $X$ in $U$. Then we have

Proposition 5.7. The condition (5.8) for a vector field $X^{a} \partial / \partial y^{a}$ tangent to $M$ is equivalent to the condition

$$
d \widetilde{X}^{\lambda} \wedge d\left(z^{1} \circ i\right) \wedge \cdots \wedge d\left(z^{n+1} o i\right)=0
$$

where $i: M \rightarrow \widetilde{M}$ is the immersion of $M$ and $\widetilde{X}^{h}=X^{a} B_{a}^{h}$.

We now assume that $M$ is a real hypersurface analytically immersed in $M$ and that $X$ is an analytic vector field in $M$. Then, as is well known, the differential equation (5.12) with unknown functions $\tilde{X}^{\lambda}\left(z^{\mu}, \bar{z}^{\mu}\right)$ has a local solution $\tilde{X}^{\lambda}$ satisfying the boundary condition

$$
\left(\tilde{X}^{\lambda} o i\right)=\tilde{X}^{\lambda}
$$

along $M$, when $X$ satisfy condition (5.13) [5]. Therefore, taking account of Proposition 5.3 and Lemmas 5.5, 5.6 and 5.7, we can prove the following proposition:

Proposition 5.8. Let $M$ be a real hypersurface analytically immersed in an almost hyperbolic Hermitian manifold $\widetilde{M}$. Then an analytic vector field $X$ in $M$ is pseudoconformal if and only if, for any point $P$ belonging to $M$, there are a neighborhood $O$ of $M$ containing $P$ and a homomorphic vector field $\widetilde{X}$ in $\widetilde{O}$ such that $X$ is the restriction of $\widetilde{X}$ to $\widetilde{O} \cap M$.

\section{Acknowledgement}

Author is thankful to the referee for his helpful comments and suggastetion towards the improvement of the paper.

\section{References}

[1] E. Cartan, Sur la gomtrie pseudo-conforme des hypersurfaces de deux variables complexes. I, Ann. Math. Pura Appl. (4) 11 (1932), 17-90 (or Oeuvres. II, 2, 1231-1304); II, Ann. Scuola Norm. Sup. Pisa, (2) 1 (1932), 333-354 (or Oeuvres. III, 2, 1217-1238).

[2] S. S. Chern and J. Moser, Real hypersurfaces in complex manifolds, Acta Math. 133 (1974), 219-271.

[3] S. Ishihara, Distributions with complex structure, Kodai Math. J., 1(1978), 164-276. 
[4] S. Sasaki, Almost contact manifolds, Part I, Lecture Notes, Tohoku University, Sendai, Japan, 1965.

[5] N. Tanaka, On the pseudo-conformal geometry of hypersurfaces of the space of $n$ complex variables, J. Math. Soc. Japan 14 (1962), 397-429.

Department of Mathematics, University of Allahabad, Allahabad-211002.

E-mail: complex_geometry@yahoo.co.in 